\title{
Usefulness of Toys in Neurodevelopmental disorders-A Survey amongst Physiotherapists
}

\author{
Rucha R Gadgil ${ }^{1}$ and Isha S Akulwar ${ }^{2 *}$ \\ ${ }^{1}$ Student of M.P.Th. in Neurosciences, Institute: K. J. Somaiya college of Physiotherapy, Mumbai, India \\ ${ }^{2}$ M.P.Th. in Neurosciences, Associate Professor in Neurosciences Physiotherapy Institute: K. J. Somaiya college of Physiotherapy, Mumbai, India
}

*Corresponding author: Isha S Akulwar, M.P.Th.Neurosciences Physiotherapy, Associate Professor, K.J. Somaiya College of Physiotherapy, Mumbai, India.

Received Date: October 01, 2019

Published Date: October 10, 2019

\section{Abstract}

Background: Neuromuscular disorders create profound movement disorders in children affecting their development physically and mentally. Considering the challenging circumstances of pediatric population with developmental disorders therapeutic toys can prove to be beneficial in therapy in achieving both functional goals and psychological goals. This study aimed to assess Physiotherapists' knowledge of use of therapeutic toys as an approach in therapy of children with developmental disabilities.

Method: A semi-structured, self-administered questionnaire prepared on Google Forms was circulated among physiotherapists having experience in pediatric physiotherapy via social media. Data analysis was done by Google spreadsheet.

Results: 102 physiotherapists responded. $99 \%$ were using toys with $80 \%$ using them constructively. 38\% reported they did not know the impairments that can be targeted and properties that could be useful. $56 \%$ said there was no evidence of toys in therapy.

Conclusion: Awareness regarding therapeutic use of toys is required in pediatric physiotherapists.

\section{Introduction}

Currently, there are more than 150 million children in the world known to have a disability [1]. Neurodevelopmental disorders are defined as a group of heterogeneous conditions characterized by a delay or disturbance in the acquisition of skills in a variety of developmental domains, including motor, social, language, and cognition with severity ranging from mild to most severe [2]. It is unknown if the child's mental delays are due to his own incapability or a merely lack of tools to help him learn and express himself. These disorders are also known to have an influence on the quality of life in children and may completely change their functional and cognitive abilities and further: life attitude [3]. Physical therapy and occupational therapy efforts in the pediatric population target reshaping of the nervous system, which is capable of reorganization and change through a variety of neural plasticity mechanisms, especially in the developing brain [4].

However, the key concerns of any therapeutic program especially in the pediatric population are maintaining patient motivation, level of engagement, and adherence. Children with neurodevelopmental delays have limited opportunities to explore their physical and social environment. These children are usually more passive than children without disabilities, when playing with objects and become more actively engaged if provided with appropriate stimuli (e.g., suitable toys, other objects and materials) that are fostered through social interactions and play [5]. Recent studies on constraint-induced movement therapy (CIMT) and robotic therapy have shown that intensive, goal-directed rehabilitation therapies substantially improve patients' motor function and their participation in the community. However, such therapies are timeintensive or institution-based, and hence costly. Thus, there is a need for an engaging, inexpensive approach to neuro-muscular therapy [6]. Similarly, new rehabilitation techniques based on recent neuro-scientific advances have been suggested to enhance recovery and to promote brain plasticity in patients following brain damage. These techniques, however, have not focused on children nor have emerging methods, capitalized upon the primary learning avenue for children - playing. Play is a child's natural need [7]. The role of play in the development of children has been extensively studied and it has already been proven that play plays an important and large part in childhood [8]. A large body of work exists to 
discuss the importance and nature of play in children and the toys that can be utilized for the same. Incorporating this into therapy especially when children with neuromuscular disorders require long term therapy, toys can play a greater role as a major adjunct to therapy. Toys can be used to help improve a child's motor, cognitive, sensory processing, communication, and play skills with the goal of enhancing their development and minimize the potential for developmental delay. Toys use during therapy can be an engaging and motivating solution to the current limitations of physical and neuro rehabilitation and may serve as an easily available, low-cost, fun, and functional option for children with special needs.

Although theoretically positive value of toys seems to be established, there has not yet been sufficient evidence focused on the use of toys in child-based clinical settings. Despite toys being commonly used in the treatment of children with developmental disabilities, research and scientific publications concerning this issue, especially in infants, are still rare. As a result, use of toys in neurodevelopmental disorders is not yet optimized. At the same time, knowledge of the use of toys in a rehabilitative and structured way for the betterment of the child is an aspect that needs to be addressed. Thus, this study aimed at exploring the knowledge of pediatric physiotherapists regarding the use of toys in therapy.

\section{Methods}

This was a qualitative study undertaken with the aim of understanding use of toys amongst Physiotherapists working with neurodevelopmentally affected children. A self-administered questionnaire was prepared on Google Forms with open and close ended questions. The questionnaire sought to obtain information about awareness of the therapeutic aspects of toys during therapy. It had 14 questions with 5 questions being open-ended. Close-ended questions had affirmative and negative options. Face validity was established from 3 subject experts having a minimum of 5 years' experience in pediatric field. The questionnaire was prepared on Google Forms and circulated on social media amongst Pediatric Physiotherapists.

Data Analysis: The close-ended data was analyzed automatically using Google spread- sheet and represented statistically using percentage. The open-ended questions were analyzed using categorization and thematic analysis. To analyze the data of the open-ended questions by categorization, it was fed into a spreadsheet and common response categories were identified (set of replies that can be grouped) and organized in a set of patterns. This sorted data was then represented visually with the help of graphs. Thematic analysis was done for the questions that had complex responses to categorize. Procedure as proposed by the wellcited article by Braun and Clarke was followed. In detail, the data corpus of the transcripts of the answers was read multiple times. An initial set of codes (open coding) was generated and applied to the texts. This process was repetitive and synthetic, that is, codes were given on a data-driven basis instead of being inspired by theory or existing coding frameworks. Next, these initial themes were discussed and were shaped into the final theme definitions.
Specifically, descriptive names were given for the themes to capture and represent the salient issues [9].

\section{Results}

102 Physiotherapists having experience of $5.1196 \pm 0.74$ (mean \pm SD) years in Pediatric Physiotherapy filled the questionnaire. 99\% responded that they use toys in their therapy with children. When asked about which toys do they use: 78.4\% responded that they use the toys available in their clinical setting while only $8.8 \%$ customize their toys according to the needs of the children. The rest of them $(10.8 \%)$ asked the parents of the children to get the toys. Regarding the goal for its use $80.2 \%$ said that they use toys working towards a therapeutic goal with a reasoning behind it as well as to maintain attention of the child, $14.9 \%$ said that they use toys only to work towards a specific goal that they have in mind and 5\% use it only to get the child to cooperate with the therapist.

While answering about the type of conditions in which toys can be used, more than $60 \%$ mentioned cerebral palsy as the only condition while $20 \%$ were of the opinion that toys can be utilized in all the neuro-developmental and pediatric neuromuscular conditions.

For the open-ended questions on the therapeutic goals that can be achieved by using toys in rehabilitation of the child-A majority of them said that use of toys has the greatest impact in treating upperlimb motor functions. With around 20\% were unable to enlist the purpose of use of toys in therapy.

The therapists that responded were unable to list out the functions that they look for when suggesting/ using toys when doing therapy. When asked about articles/evidence which supports the use of toys in Physiotherapy of children, 32.4\% agreed that they have come across studies on importance of use of toys in pediatric therapy while $67.7 \%$ said that they have never searched for the same. $56 \%$ of the respondents agreed that there is a dearth of appropriate toys in India, 35\% were unaware as to what to look for while only $7.9 \%$ therapists said their availability is adequate.

While answering about what kind of toys should be available in India to aid rehabilitation goals, $58 \%$ were unable to specify their requirement; $16 \%$ wanted more variety of sensory toys, $15 \%$ wanted more freedom in customizing toys and $11 \%$ were satisfied with the current availability. When asked about the sources of the toys used, $42.4 \%$ said that they used whatever toys the parents of the child had, $40 \%$ said that they use generic toys stores, $17.6 \%$ said that they have a resource person/ source in India who helped them avail the necessary toys according to their specifications. While all the respondents agreed to the need for customizing toys, $54 \%$ agreed that it was easier to customize toys when using for therapeutic goals. $98 \%$ of the therapists were in agreement that use of toys in therapy will definitely have a positive effect on the rehabilitative progress of the child whereas remaining $2 \%$ were uncertain.

A common theme arose when asked for suggestions/opinions regarding use of toys in neurodevelopmental disorders: The 
responders wanted that constructive use of toys in rehabilitation should be taught as a part of curriculum to students of physiotherapy. They also agreed to dearth in evidence and suggested more studies to be done regarding the same.

\section{Discussion}

Pediatric neurological rehabilitation is a challenge. It is difficult maintaining compliance of a growing child with present therapeutic techniques. Pediatric physical therapy is different from adult therapy in that younger children typically cannot (or may not be willing to) follow direct instructions required of a therapy routine. Thus, therapists typically have to resort to incorporating ingenious ways of play to provide an engaging and motivational intervention that may enhance the child's participation in the therapy session. Motivation, play and sensitivity in interaction are categorized as important elements in physical therapy [10].

Piaget's book "Play, dreams, and imitation in childhood" is one of the earlier references showing the importance of play in the learning of cognitive, social, and physical skills [11]. It suggests that play is useful for a variety of reasons, including helping to develop motor skills and spatial abilities. However, play is primarily referred to as a developmental domain and a context in which intervention occurs [12]. Play therapy is a therapeutic and psychological intervention that incorporates the child's physical abilities, cognitive functioning levels and emotional needs using both directed and non-directed play. Play during therapy with use of toys can bring experiences universal to childhood. Toys have an important role in the developmental context of normal children in terms of the cognitive, social, motor, psychological and sensory aspects. The use of toys is also becoming a pleasant and motivational tool for supporting rehabilitation and permeating a diversity of therapies of infant-juvenile patients. Selecting an appropriate toy geared towards learning and considering the physical and developmental needs of a child with neurological condition would enhance therapeutic outcomes. Use of toys during therapy would provide additional opportunities to develop their physical and mental abilities and explore physical as well as social environment.

It is important to mention that none of the therapists were aware of the use of toys for assessment of children. Spontaneous play with toys can be early diagnostic tool for assessment interaction in autistic children, taking into consideration eye contact, touch, manipulation, and posture [13]. Toy/object exploration can also be observed in play if children with Down syndrome [14].

Toys for children with motor deficits support therapy and make easier child-therapist interaction. Toys act as a medium through which the child communicates, learns, self-discovers, shares experiences and forms a trusting therapeutic relationship, thus serve simply as a bridge to therapy. 'They can play crucial role in infants where motivation and stimulation of the young patient is hard to achieve. Thus therapy of young children should be shaped as toy play, promoting requested child behaviors, including more advanced toy play. Intelligent multifunctional toys are the next step toward increased use of toys in the therapy of older children with developmental disorders. Use of toys would engage the child encouraging active participation in therapy and thus, may enhance the therapeutic intervention. Also, reduced playfulness in children with developmental disabilities may be treated using toys during therapy. Thus, use of toys may address the personal and social environmental factors in therapy context from the ICF perspective.

In a study conducted by Peter Fikar et al, it was found that therapeutic toys are often accepted for their open-endedness and versatile applicability, meeting individual needs and making therapeutic sessions both enriching and fun for the children [15]. Use of toys can thus improve the quality of children's experiences during therapy. Studies have shown that motivation through the use of toys also plays an important role towards maintaining the attention of the child in doing a long, arduous and repetitive tasks5. Therapists participating in this study also supported that majority of them plan the use of toys in their therapy session.

Children with neurocognitive impairments may experience decreased functioning in various domains including attention, self-awareness, memory, reasoning and judgment. Children with disabilities require additional supports to help them anticipate events and understand what behaviors are expected of them. Additional structures such as instructions, cues, prompts and feedback can be easily integrated with use of appropriate toys during therapy. To counter some concerns with respect to findings that state task-specificity and goal- orientedness are crucial aspects in the treatment of children versus passive training for motor learning (Papavasiliou 2009), it becomes crucial to incorporate the use of interactive toys and play in pediatric therapy setting. However, utilization of toys when dealing with neurodevelopmental disorders is still an unchartered territory as of now. Though studies have been done with the use of toys in therapy; awareness about the same in developing nations is on the lower spectrum; which is an issue that needs to be addressed. The results of a study by Lifter et al in 2011 showed the positive use and effect of play in therapeutic intervention scenarios [16], for children with developmental delays. The responders in this study also agreed to positive effect of toys in pediatric interventions but felt that the knowledge regarding proper use is lacking.

Use of toys as a part of therapy should be taught from the grassroot level i.e. from graduation schools itself so as to promote their efficient and qualitative use. Though therapists are aware about the use of toys in therapy, they need to be educated more on functions to look for in a toy which will be age appropriate and goal-oriented while being used in therapy. Each toy designed has a specific objective in mind and allows for well-defined aims5. Educating the therapists about therapeutic properties of the toys will assist them in making decisions about toy selection and for effective patient-tailored approach. The choice of toys might determine, in advance, and/or restrain the therapeutic outcome for the targeted impairment. 
It is important to mention that toys are not categorized by disability but are labeled with age. Adding symbols to the toys based on different skills such as motor, tactile, cognitive, visual, auditory, language, social, etc. will allow therapists and parents to quickly find what toys are most appropriate for the individual needs of the child. Several types of toys already exist, ranging from the simpler ones operated by children with motor impairments for use in manipulation-based play to the advanced ones integrating play with autonomous robot toys for engaging children with neurodevelopmental disorders.

Though variety of toys are usually available in generic stores customization is required in the context of special needs of developmentally disabled children. Identified flaws with the original toys are that they are too small and/or complex- larger, visually simpler, easier to grab toys can be some of the modifications required. When designing and creating toys for these children it is important to find a balance between providing for his developmental level, physical needs and functional skills required in daily life. Paying attention to one delayed skill is not reasonable. Balanced simultaneous development of all skills (motor, cognitive, social) can play crucial role for achievement of subsequent developmental milestones. Along with targeting physical impairments, selecting an appropriate toy which stimulates visual, auditory and other development and is conducive to play is important.

The availability of therapeutic toys in India is a rising query and though new avenues are arising every day, the financial aspect also needs to be taken care of. The requirement of toys for therapeutic purposes and their standardized availability across the country is disproportionate with therapists using toys. The therapists are using toys according to their availability as opposed to as per the need in achieving a particular goal, which is something that needs to be changed. To address this issue toy library concept can be used for children with developmental disabilities. Innovations involving specifically designed toys and technology should be encouraged on a larger scale to provide a multidisciplinary approach and better results in a pediatric programme4 for e.g. Pervasive play [17]. There is a dearth of supporting literature regarding the same.

Whether using commercially designed or customized ones, toys can serve to enhance outcomes of therapeutic interventions in pediatric setting when used appropriately. However, avenues for customization of toys as per the therapists' view are severely lacking. The therapists should be made aware about the constructive, effective and efficient use of the toys targeting identifiable domains in pediatric therapy. Use of toys therapeutically as monitors and sources of stimulation may shape new mixed intervention strategies.

\section{Conclusion}

Results of this study bring up an observation that though toys are being widely used as a part of therapy in neurodevelopmental disorders, their use is haphazard and without a goal. This study highlighted lack of awareness among practicing physiotherapists about varied therapeutic uses of toys in pediatric rehabilitation. It is also difficult to find a general or holistic guidance, or any concrete entry points to promote this endeavor. The dearth of scientific literature in usefulness of toys in rehabilitation, proved to be a necessity for this study to establish a starting point towards educating pediatric therapy and finding an innovative treatment approach in the ever-changing field. Further studies can be carried out on using particular toys in conjunction with therapy and finding their effects on impairments in particular neurodevelopmental disorders.

\section{Acknowledgement}

Authors would like to thank study participants and Principal of K. J. Somaiya college of Physiotherapy, Mumbai for their valuable contribution.

\section{Conflict of Interest}

No conflict of interest.

\section{References}

1. Howard AM (2013) Robots learn to play: Robots emerging role in pediatric therapy. FLAIRS 2013 - Proceedings of the 26th International Florida Artificial Intelligence Research Society Conference: 3-8.

2. Jeste $S$ (2015) Neurodevelopmental behavioral and cognitive disorders. CONTINUUM (MINNEAP MINN): 690-714.

3. Mikołajewska E, Komendziński T, Dreszer J, Bałaj B, Mikołajewski D (2015) Role of toys in the development and rehabilitation of children with developmental disorders. Journal of Education, Health and Sport 5(4): 224-228.

4. Wilcox B, Wilkins M, Basseches B, Schwartz JB, Kerman K, et al. (2016) Joint-Specific Play Controller for Upper Extremity Therapy: Feasibility Study in Children with Wrist Impairment. Phys Ther 96(11): 1773-1781.

5. Fikar Peter, Güldenpfennig Florian, Ganhör, Roman (2018) The Use(fulness) of Therapeutic Toys: Practice-derived Design Lenses for Toy Design: 289-300.

6. Aisen ML, Kerkovich D, Mast J, Mulroy S, Wren TA, et al. (2011) Cerebral palsy: clinical care and neurological rehabilitation. Lancet Neurol 10: 844-852.

7. Winnicott D (1971) Playing and Reality.

8. Linder TW (1993) Transdisciplinary play-based assessment: A functional approach to working with young children ( $2^{\text {nd }}$ ed.). Baltimore MD: Brookes, USA.

9. Braun Virginia, Clarke Victoria (2008) Using Thematic Analysis in Psychology. Qualitative Research in Psychology 3: 77-101.

10. Blanchard Y, Oberg GK (2015) Physical therapy with newborns and infants: applying concepts of phenomenology and synactive theory to guide interventions. Physiother Theory Pract 31(6): 377-381.

11. Piaget J (1951) Play, Dreams and Imitation in Childhood.

12. Lifter Karin, Mason Emanuel J, Barton Erin E (2011) Children's Play: Where We Have Been and Where We Could Go. Journal of Early Intervention 33(4): 281-297.

13. Giannopulu I, Pradel G (2010) Multimodal interactions in free game play of children with autism and a mobile toy robot. Neuro Rehabilitation 27(4): 305-311.

14. Ruskin EM, Mundy P, Kasari C, Sigman M (1994) Object Mastery Motivation of Children with Down Syndrome. Am J Ment Retard 98(4): 499-509.

15. Florian Güldenpfennig, Peter Fikar, and Roman Ganhör (2018) Interactive and Open-Ended Sensory Toys: Designing with Therapists and Children 
for Tangible and Visual Interaction. In International Conference on Tangible, Embedded and Embodied Interactions, Stockholm: 451-459.

16. Lifter Karin, Foster Sanda, Suzanne MS (2011) Overview of Play: Its Uses and Importance in Early Intervention/Early Childhood Special Education. Infants \& Young Children 24(3): 225-245.
17. Ahn June, Bonsignore Elizabeth, Hansen Derek, Kraus Kari, Neustaedter Carman (2016) Pervasive Play. The 2016 CHI Conference Extended Abstracts: $3317-3324$. 